\title{
APPROXIMATION OF REAL CONTINUOUS FUNCTIONS ON LINDELÖF SPACES ${ }^{1}$
}

\author{
ANTHONY W. HAGER ${ }^{2}$
}

Throughout, topological spaces are assumed to be completely regular and Hausdorff. $C(X)$ is the set (or ring) of real-valued continuous functions on the space $X ; C^{*}(X)$ is the subset (or subring) of bounded functions. The subset $B$ of $C(X)$ is said to separate points and closed sets of $X$ if given a closed set $F$ and a point $x \notin F$, there is $f \in B$ with $f(x) \notin \operatorname{cl}(f(F))$.

We state the principal result.

1.4 TheOREM. Let $X$ be a space with the Lindelöf property, and $A$ a subring of $C^{*}(X)$ containing the rational constants and separating points and closed sets of $X$. Then, each function in $C(X)$ is the pointwise limit of a sequence from $A$; if $A$ is also a lattice, then each nonnegative function in $C(X)$ is the pointwise limit of an increasing sequence from $A$.

The conclusion of 1.4 asserts that $A$ is "sequentially dense" in $C_{p}(X)(=C(X)$ with the topology of pointwise convergence). When $A$ is a lattice, the conclusion can be combined with Dini's Theorem to obtain sequential density in $C_{c}(X)(=C(X)$ with the compact-open topology). This allows the following estimate of the "sequential density character" of, e.g., $C_{c}(X)$, for infinite Lindelöf $X$ : it does not exceed the weight of $X$; it follows that the cardinal of $C(X)$ does not exceed (weight $X$ ) ${ }^{N_{0}}$, and with a result of Kruse, equality holds.

1. The main results. The method of proof of 1.4 can be used to obtain some similar theorems $(\S 2)$, so we proceed somewhat more generally.

Suppose that $X$ is an arbitrary space, and that $A$ is a subring of $C^{*}(X)$ which contains the rational constants and separates points and closed sets in $X$. Let $A_{1}$ be the smallest subring of $C(X)$ which contains $A$ and is closed under uniform convergence and inversion of functions without zeros.

Presented to the Society, April 5, 1969 under the title Approximation of continuous functions on Lindelö spaces; received by the editors September 6, 1968.

${ }^{1}$ Research partially supported by the National Science Foundation under grant GP-7455, at the University of Rochester.

2 I am pleased to thank Arthur Stone for several valuable conversations, W. W. Comfort for several valuable conversations and for the examples 3.4(i) and 3.4(ii), and Frank Anderson for pointing out an error in an earlier version of this paper. 
1.1 Proposition. Each function in $A_{1}$ is the pointwise limit of a sequence from $A$; if $A$ is a lattice, then each nonnegative function in $A_{1}$ is the pointwise limit of an increasing sequence from $A$.

Proof. A description of $A_{1}$ is needed. For $B C C(X)$, let ul $B$ denote the collection of Uniform Limits of sequences from $B$. Then $A_{1}=\mathrm{ul}\{f / g: f, g \in \mathrm{ul} A$ and $g$ has no zeros $\}[\mathrm{H}, 2.1$ and 3.1]. (In the results in $[\mathrm{H}]$, the ring of functions extendable over a certain compactification of $X$ replaces ul $A$. That ul $A$ is one of these can be seen by embedding $X$ into a cube whose factors are indexed by $A\left[\mathrm{~K}_{1}, \mathrm{p}\right.$. 116]. An application of the Weierstrass-Stone Theorem $\left[K_{1}\right.$, p. 244] establishes that ul $A$ is exactly the set of functions extendable over the closure of $X$ in the cube.) The computations below will yield 1.1. We shall need to know that ul $A$ is a lattice, a ring, contains all real constants, and if $f \in \mathrm{ul} A$ with $f \geqq a>0$, then $1 / f \in u l A$. These are easily proved, with the possible exception of the first; for this, see [GJ, 16.2].

We first show that the assertions of 1.1 hold for the functions $\mathrm{f} / \mathrm{g}$ in the description of $A_{1}$.

Consider $g \in \mathrm{ul} A$ with $g(x)>0$ for each $x \in X$. With $g_{n}=$ $\max \{g, 1 / n\},\left(g_{n}\right)$ decreases and converges uniformly to $g$. Now, $1 / g_{n} \in \mathrm{ul} A$, so there is a sequence $\left(g_{k}^{(n)}\right)$ from $A$ which converges uniformly to $1 / g_{n}$. We arrange it so that $1 / g_{n}(x)-1 / 2^{k} \leqq g_{k}^{(n)}(x) \leqq 1 / g_{n}(x)$ $-1 / 2^{k+1}$ (for all $x \in X$ ); it follows that $\left(g_{n}^{(n)}\right)$ increases and converges pointwise to $1 / g$.

Now consider an $f / g$. We assume $g(x)>0$ for all $x$ (by replacing $g$ by $g^{2}$ and $f$ by $f g$, if necessary), and choose $\left(g_{n}^{(n)}\right)$ as in the preceding paragraph. Let $\left(f_{n}\right)$ be a sequence from $A$ converging uniformly to $f$. Then $\left(f_{n} g_{n}^{(n)}\right)$ converges pointwise to $f / g$.

If $A$ is a lattice and $f / g \geqq 0$, we assume that $f \geqq 0$ and that $g(x)>0$ for all $x$ (by replacing $f$ by $|f|$ and $g$ by $|g|$, if necessary). Pick $\left(g_{n}^{(n)}\right)$ as before. Let $\left(f_{n}\right)$ be a sequence from $A$ which increases and converges uniformly to $f$; we assume each $f_{n} \geqq 0$ (by replacing $f_{n}$ by $f_{n} \vee 0$, if necessary). Then $\left(f_{n} g_{n}^{(n)}\right)$ increases and converges uniformly to $f / g$.

Observe that in each of the two preceding paragraphs, if $|f / g| \leqq M$, then upon choosing $\left(f_{n}\right)$ so that $\left|f_{n}-f\right| \leqq M \cdot(1 / n)$, and noticing that $\left|g_{n}^{(n)}\right| \leqq n$, we have

$$
\begin{aligned}
\left|f_{n} g_{n}^{(n)}\right| & \leqq\left|f_{n} g_{n}^{(n)}-f g_{n}^{(n)}\right|+\left|f g_{n}^{(n)}\right| \\
& \leqq\left|f_{n}-f\right| \cdot\left|g_{n}^{(n)}\right|+|f / g| \leqq 2 M
\end{aligned}
$$

Now let $h \in A_{1}$, so that $h$ is the uniform limit of a sequence $\left(h_{n}\right)$ 
of $f / g$ 's and in such a way that for $n>1$ each function $u_{n}=h_{n}-h_{n-1}$ is bounded, say $\left|u_{n}\right| \leqq M_{n}$, with $\sum M_{n}<\infty$. Each $u_{n}$ is an $f / g$, and hence the pointwise limit of a sequence $\left(u_{k}^{(n)}\right)$ from $A$; by the preceding paragraph, we take $\left|u_{k}^{(n)}\right| \leqq 2 M_{n}$ (for each $k$ ). With $\phi_{n}=u_{n}^{(2)}$ $+\cdots+u_{n}^{(n)}, \phi_{n} \in A$. We show below that $\left(\phi_{n}\right)$ converges pointwise to $h-h_{1}$. Knowing this, we take a sequence $\left(h_{n}^{(1)}\right)$ from $A$ which converges pointwise to $h_{1}$, and $\left(\phi_{n}+h_{n}^{(1)}\right)$ is a sequence from $A$ converging pointwise to $h$.

The modifications necessary to get an increasing sequence, in case $A$ is a lattice and $h \geqq 0$, are as follows. Take $\left(h_{n}\right)$ increasing, and $h_{1} \geqq 0$. (The first is always possible, and the second is possible whenever $h \geqq 0$, because $A_{1}$ is always a lattice. These are easy to show.) Thus each $u_{n} \geqq 0$, and we may and do take each $u_{k}^{(n)} \geqq 0$ and $\left(u_{k}^{(n)}\right)$ increasing; this makes $\left(\phi_{n}\right)$ increase. Upon taking $\left(h_{n}^{(1)}\right)$ increasing, the sequence $\left(\phi_{n}+h_{n}^{(1)}\right)$ increases.

Finally, let $\epsilon>0$ be given. Choose $m$ with $\sum_{n>m} 2 M_{n}<\epsilon / 3$. Let $x \in X$, and $r>m$. Then

$$
\begin{array}{r}
\left|\left(h(x)-h_{1}(x)\right)-\phi_{r}(x)\right|=\left|\sum_{n=2}^{\infty} u_{n}(x)-\phi_{r}(x)\right| \leqq\left|\sum_{n=2}^{m}\left(u_{n}(x)-u_{r}^{(n)}(x)\right)\right| \\
+\left|\sum_{n=m+1}^{r} u_{r}^{(n)}(x)\right|+\left|\sum_{n=m+1}^{\infty} u_{n}(x)\right| .
\end{array}
$$

For large enough $r$ (depending on $x$ ) the first term is $<\epsilon / 3$; for any $r$, the second and third terms are each $<\epsilon / 3$. Thus $\phi_{r}(x) \rightarrow\left(h(x)-h_{1}(x)\right)$. (This argument is based on one in [N, p. 132].)

The proof of 1.1 is complete.

At this point, the proof of the approximation theorem for Lindelöf spaces can be completed with a reference: if $X$ is Lindelöf, then (for any $A_{1}$ as in 1.1) $A_{1}=C(X)$, by a result due to M. Henriksen and D. G. Johnson [HJ, 5.4], in an algebraic setting, and later and independently to $S$. G. Mrówka $\left[\mathbf{M}_{2}, 4.6\right]$. As is made clear in both papers, the question of equality of $A_{1}$ and $C(X)$ involves the "zero-sets"; we discuss this in a little more detail.

The zero-set of the function $f$ is the set $Z(f)=\{x: f(x)=0\}$. It was first noticed by Isbell [I, $1.31(\mathrm{c})]$ that $A_{1}=C(X)$ if the zero-sets of the functions in $C(X)$ and in $A_{1}$ coincide. (Proofs appear in $[\mathrm{HJ}$, 5.2], $\left[\mathbf{M}_{2}, 4.4\right]$ and $[\mathrm{H}, 3.9]$.) From the description of $A_{1}$ quoted in the proof of 1.1 , it follows easily that the zero-sets of functions from $A_{1}$, and from ul $A$, coincide. (And, if $A$ is a lattice, and $f \in u l A$, 
then there are $f_{1}, f_{2}, \cdots \in A$ with $Z(f)=\bigcap Z\left(f_{n}\right)$.) Thus, with notation as in 1.1

$1.2 A_{1}=C(X)$ if (and only if) the zero-sets of functions in $C(X)$ are zero-sets of functions in ul $A$.

For Lindelöf spaces, the following explains why $A_{1}=C(X)$.

1.3 Proposition (Jerison, [HJ, 5.3]). Let $X$ be a Lindelöf space, and $B \subset C^{*}(X)$ a ring containing constants, separating points and closed sets, and closed under uniform convergence. Then the zero-sets of functions in $C(X)$ are zero-sets of functions in $B$.

Proof. (Included because [HJ, 5.3] is stated somewhat differently.) Let $f \in C(X)$. For each $x \notin Z(f)$, choose $f_{x} \in B$ with $0 \leqq f_{x} \leqq 1, f_{x}(x)>0$, and $f_{x}(Z(f))=\{0\}$. Since $X$ is Lindelöf, so is $X-Z(f)$ (being an $F_{\sigma}$ ), and there are $x_{1}, x_{2}, \cdots$ with $X-Z(f)=\mathrm{U}\left(X-Z\left(f_{x_{i}}\right)\right)=$ $X-Z\left(\sum 2^{-i} f_{x_{i}}\right)$. The series converges uniformly, so $\sum 2^{-i} s_{x_{i}} \in B$.

Theorem 1.4 follows immediately from 1.1, 1.2, and 1.3.

1.5 Examples. (i) In 1.4, the hypothesis that $X$ have the Lindelöf property is not superfluous. Let $X$ be the real line with the discrete topology, and $A$ the ring of bounded Baire functions on the real line [N, Chapter XV]. The pointwise limit of a sequence from $A$ is again a Baire function; since there are only $c$ Baire functions, not every function in $C(X)$ is the pointwise limit of a sequence from $A$.

(ii) In 1.4, the hypothesis that $A$ separates points and closed sets cannot be replaced by the hypothesis that $A$ separates points (though the latter suffices that $A$ be dense in $\left.C_{p}(X)\right)$. We shall exhibit a pair of spaces $(X, Y)$ such that $X$ has the Lindelöf property, $Y$ is a $P$-space (i.e., $G_{\delta}$ 's are open), and there is a continuous one-to-one map $\phi$ of $X$ onto $Y$ which is not a homeomorphism. Given such a pair, let $A=\left\{f \circ \phi: f \in C^{*}(Y)\right\}$. Clearly, $A$ is a subring of $C^{*}(X)$ which separates points but not points and closed sets. Each pointwise limit of a sequence from $A$ lies in $\{f \circ \phi: f \in C(Y)\}$ (which is not all of $C(X)$ ). For, if $\left(h_{n}\right)$ is a sequence from $A$ converging pointwise on $X$ to $h \in C(X)$, then $h_{n}=f_{n} \circ \phi$, and $\left(f_{n}\right)$ converges pointwise on $Y$ to some function $f$. Because $Y$ is a $P$-space, $f \in C(Y)$, and $h=f \circ \phi$ follows easily.

Such a pair of spaces is constructed as follows. Let $D_{1}$ and $D_{2}$ be disjoint uncountable sets, $p_{1}$ and $p_{2}$ two different objects with $p_{i} \notin D_{i}$. Let $Y$ be the disjoint union of the spaces $D_{i} \cup\left\{p_{i}\right\}$, where each point of $D_{i}$ is isolated, and each neighborhood of $p_{i}$ has countable complement in $D_{i}$. Let $X$ be the space $D_{1} \cup D_{2} \cup\left\{p_{1}, p_{2}\right\}$ with to- 
pology: each point of $D_{1} \cup D_{2} \cup\left\{p_{1}\right\}$ is isolated, and each neighborhood of $p_{2}$ has countable complement. The map $\phi$ is the identity.

2. We note two easy applications of the results of $\$ 1$.

2.1 Theorem. Let $M$ be a metrizable space, and $X$ an arbitrary subspace of $M$. Then, each nonnegative function in $C(X)$ is the pointwise limit of an increasing sequence of functions with continuous extension over $M$.

Proof. Let $A$ be the collection of functions in $C(X)$ with continuous extension over $M$. Then $A=\mathrm{ul} A$, and is a lattice. Because $M$ and $X$ are metrizable, a zero-set of $X$ is just a closed set in $X$, which extends to a closed set of $M$, which is a zero-set of $M .1 .2$ and 1.1 now apply.

Now, let $X=\prod_{\lambda \in \Lambda} X_{\lambda}$ be the topological product of spaces $X_{\lambda}$; the index set $\Lambda$ is arbitrary. By a "countable subproduct of $X$ " is meant a space of the form $\prod_{\lambda \in \Lambda_{0}} X_{\lambda}$, where $\Lambda_{0}$ is a countable subset of $\Lambda$. Let $\otimes_{\lambda \in \Lambda} C^{*}\left(X_{\lambda}\right)$ be the subring of $C^{*}(X)$ generated by the functions of one variable, i.e., by the functions of the form $f \circ \Pi_{\lambda}$, where $\Pi_{\lambda}$ is the projection of $X$ onto $X_{\lambda}$, and $f \in C^{*}\left(X_{\lambda}\right)$.

2.2 THEOREM. If each countable subproduct of $X=\prod_{\lambda \in \Delta} X_{\lambda}$ has the Lindelöf property, then each function in $C(X)$ is the pointwise limit of a sequence from $\otimes_{\lambda \in \Lambda} C^{*}\left(X_{\lambda}\right)$.

Proof. Let $f \in C(X)$. By [E, Theorem 1], there is a countable subproduct $X_{0}=\prod_{\lambda \in \Lambda_{0}} X_{\lambda}$ and $g \in C\left(X_{0}\right)$ with $f=g \circ \Pi_{0}$, where $\Pi_{0}$ denotes projection onto $X_{0}$. Applying 1.4 on the space $X_{0}, g$ is the pointwise limit of a sequence $\left(g_{n}\right)$ from $\otimes_{\lambda \in \Lambda_{0}} C^{*}\left(X_{\lambda}\right)$. Clearly, $g_{n} \circ \Pi_{0} \in \otimes_{\lambda \in \Lambda} C^{*}\left(X_{\lambda}\right)$, and $\left(g_{n} \circ \Pi_{0}\right)$ converges pointwise to $f$.

2.3 Remarks. (i) We comment on when the hypothesis to 2.2 holds, i.e., when the product of a countable family of spaces $\left\{X_{n}\right\}$ has the Lindelöf property. It does when each $X_{n}$ has a countable base, of course. By applying $[\mathrm{HIJ}, 2.3]$, it can be shown that $\prod X_{n}$ is Lindelöf if each $X_{n}$ is either $\sigma$-compact or Lindelöf and complete in the sense of Cech (i.e., a $G_{\delta}$ in $\beta X$ ). The latter is in [E, p. 223]; the above "proof" seems more direct. I am not aware of the result for $\sigma$-compact spaces in the literature; it was known previously to A. $\mathrm{H}$. Stone.

(ii) In $[\mathbf{R}]$, Rubin has obtained a conclusion similar to that of 2.2 under the much stronger hypothesis that $X$ be $\sigma$-compact and locally compact. The conclusions of the results differ in that Rubin shows that if the function to be approximated is nonnegative, then 
the approximating sequence can be chosen so that each $f \circ \Pi_{\lambda}$ which appears in each term of the sequence is nonnegative also. Aside from this latter twist, Rubin's Theorem is almost immediate from the Stone-Weierstrass Theorem for uniform convergence on compacta [K, p. 244] (and this proof requires only $\sigma$-compactness).

(iii) A good question is whether the conclusion to Theorem 2 holds under the hypothesis that each finite subproduct of $X$ has the Lindelöf property. (The Engelking factorization theorem used in the proof is valid in this case.) It would seem difficult to find a counterexample. To my knowledge, there is just one example known of a countable family of spaces, whose product is not Lindelöf, but each of whose finite subproducts is. The example is due to E. Michael, and uses the continuum hypothesis. (Footnote 4 of $\left[\mathbf{M}_{1}\right]$, replacing the irrationals by the homeomorphic product of countably many countable discrete spaces.) However, for $X$ this product, the conclusion to 2.2 holds. (This is not obvious.)

3. Sequential density character of $C_{c}(X)$. We shall use 1.4 to estimate the least cardinal number of a sequentially dense subset of $C_{c}(X)$ (i.e., a subset such that for each function $f$ in $C(X)$ there is a sequence from the subset converging to $f$ in the compact-open topology). We call this number the sequential density character of $C_{c}(X)$ and denote it $s \delta C_{c}(X)$. (Remarks similar to those below apply to "s $\delta C_{p}(X)$ ". This does not exceed $s \delta C_{c}(X)$, by $\left[\mathrm{K}_{1}, \mathrm{p} .222\right]$. We shall say no more about it.)

In the space $C_{c}(X)$, convergence is uniform convergence on compact sets $\left[K_{1}\right.$, p. 230]. Dini's Theorem $\left[K_{1}\right.$, p. 239] asserts that on a compact set, a pointwise convergent increasing sequence with continuous limit converges uniformly.

3.1 Proposition. Under the hypotheses of 1.4 , if $A$ is a lattice, then $A$ is sequentially dense in $C_{c}(X)$.

Proof. Let $f \in C(X)$. Write $f=f \bigvee 0-(-f) \bigvee 0=f^{+}-f^{-}$. By 1.4 and the remarks above, there are sequences $\left(g_{n}\right)$ and $\left(h_{n}\right)$ from $A$, with $\left(g_{n}\right)$ converging to $f^{+}$, and $\left(h_{n}\right)$ converging to $f^{-}$, uniformly on compact sets. Then, $\left(g_{n}-h_{n}\right)$ converges to $f$, uniformly on compact sets, hence in $C_{c}(X)$.

The weight of a space is the least cardinal number of an open base.

3.2 Theorem. For infinite Lindelöf $X, s \delta C_{c}(X) \leqq$ weight $X$.

Proof. Let $X$ be infinite and Lindelöf, and let $\beta$ be a base with card $B=$ weight $X$. Assume $X \in B$. To each pair $(U, V)$ with $U, V \in \mathbb{B}$ 
and $\bar{U} \subset V$, assign a function $f_{(U, V)} \in C(X)$ with $0 \leqq f_{(U, V)} \leqq 1, f(\bar{U})$ $=\{1\}$ and $f(X-V)=\{0\}$. ( $X$ is normal $\left[\mathrm{K}_{1}\right.$, p. 113], so such a function exists by Urysohn's Lemma.) Let $A$ be the smallest subset of $C^{*}(X)$ which is a ring and lattice, and contains all rational multiples of the $f_{(U, V)}$ 's. It is easy to map the set of all finite subsets of these rational multiples onto $A$, so that card $A=\operatorname{card}\left\{f_{(U, V)}\right\}=$ card $B$. $A$ is sequentially dense in $C_{c}(X)$, by 3.1 , and $s \delta C_{c}(X) \leqq$ weight $X$ follows.

3.3 Theorem. If $X$ is Lindelöf, and has at least two points, then card $C(X)=(\text { weight } X)^{\aleph}$.

Proof. For finite $X$, both numbers are $c$. Otherwise, by 3.2, choose $A$ sequentially dense in $C_{c}(X)$ with card $A \leqq$ weight $X$. The number of functions in $C(X)$ cannot exceed the number of sequences from $A$, i.e., card $C(X) \leqq(\operatorname{card} A) \aleph_{0}$, and card $C(X) \leqq(\text { weight } X)^{\aleph_{0}}$ follows. To show the opposite inequality, we consider $\beta X$, the StoneCech compactification of $X[\mathrm{GJ}]$. Clearly, card $C(X)=$ card $C^{*}(X)$ $=$ card $C(\beta X)$, and weight $X \leqq$ weight $\beta X$. We show that if $Y$ is any compact space, then (weight $Y)^{\aleph_{0}} \leqq \operatorname{card} C(Y)$. If $A$ is sequentially dense in $C_{c}(Y)$ (i.e., dense in the topology of uniform convergence), then the sets of the form $\{y \in Y: a<f(y)<b\}$, where $f \in A$ and $a, b$ are rational, form an open base for $Y$. That is, weight $Y \leqq s \delta C_{c}(Y)$ (for compact $Y$; compare 3.4(ii), below). Equipping $C(Y)$ with the supremum norm, we have a Banach space, and $s \delta C_{c}(Y)$ is what is called ind $C(Y)$ in $\left[\mathrm{K}_{2}\right.$, Lemma 2]; this result asserts that $\left(s \delta C_{c}(Y)\right)$ so $=$ card $C(Y)$.

3.4 Examples. (i) In 3.2 and 3.3, the hypothesis that $X$ have the Lindelöf property cannot be omitted. With $X$ the discrete reals, weight $X=c$ (so (weight $X$ ) $\aleph_{0}=c s_{0}=c$ ) and card $C(X)=2^{c}$. If $A$ is sequentially dense in $C_{c}(X)$, then $2^{c} \leqq($ card $A)$ so; this precludes card $A \leqq c$.

(ii) There is a Lindelöf space $X$ with $s \delta C_{c}(X)<$ weight $X$. Choose $q \in \beta N-N(N$ is countable discrete $)$ and let $X=N \cup\{q\}$. Weight $X>\boldsymbol{\aleph}_{0}$ [G J, 4M.2], while it is easy to see that $s \delta C_{c}(X)=\boldsymbol{\aleph}_{0}$. (In fact, $C_{c}(X)$ is metrizable and separable [W, p. 271].)

(iii) For $X$ countable discrete, $s \delta C_{c}(X)=$ weight $X$.

Finally, by the method of 3.2 and 2.2 , we can estimate $s \delta C_{c}(X)$ for certain products $X$. We omit the proof.

3.5 TheOREM. Let $X=\prod_{\lambda \in \Lambda} X_{\lambda}$ have each countable subproduct Lindelöf. Then $s \delta C_{c}(X) \leqq(\operatorname{card} \Lambda) \cdot \sum\left\{s \delta C_{c}\left(X_{\lambda}\right): \lambda \in \Lambda\right\} \leqq(\operatorname{card} \Lambda)$ - $\sum\left\{\right.$ weight $\left.X_{\lambda}: \lambda \in \Lambda\right\}$. 
Added In Proof: The inequality card $C(X) \leqq$ (weight $X$ ) so $_{\text {(for }}$ $X$ Lindelöf with at least two points), which is that part of 3.3 which uses the methods of this paper, can be given an easy direct proof by mapping $C(X)$ one-to-one into the set of sequences of countable covers of $X$ with members from a base of cardinal weight $X$.

Incidentally, from 3.3 it follows that for $X$ Lindelöf, (Weight $X$ ) $)_{0}$ $=($ weight $\beta X) \aleph_{0}$. Example 3.4 (i) shows that this equality can fail when $X$ is not Lindelöf.

\section{REFERENCES}

[E] R. Engelking, On functions defined on Cartesian products, Fund. Math. 59 (1966), 221-231.

[GJ] L. Gillman and M. Jerison, Rings of continuous functions, Van Nostrand, Princeton, N. J., 1960.

[H] A. W. Hager, On inverse-closed subalgebras of $C(X)$, Proc. London Math. Soc. (to appear.)

$[\mathrm{HJ}] \mathrm{M}$. Henriksen and D. G. Johnson On the structure of a class of archimedean lattice-ordered algebras, Fund. Math. 50 (1961), 73-94.

[HIJ] M. Henriksen, D. G. Johnson and J. R. Isbell, Residue class fields of latticeordered algebras, Fund. Math. 50 (1961), 107-117.

[I] J. R. Isbell, Algebras of uniformly continuous functions, Ann. of Math. 68 (1958), 96-125.

[K $\left.{ }_{1}\right]$ J. L. Kelley, General topology, Van Nostrand, Princeton, N. J., 1955.

$\left[\mathrm{K}_{2}\right]$ Arthur H. Kruse, Badly incomplete normed linear spaces, Math. Z. 83 (1964), 314-320.

[M $\mathbf{M}_{1}$ E. Michael, The product of a normal space and a metric space need not be normal, Bull. Amer. Math. Soc. 69 (1963), 375-376.

$\left[\mathrm{M}_{2}\right]$ S. G. Mrowka, On some approximation theorems, Nieuw Arch. Wisk. 16 (1968), 94-111.

$\left[\mathrm{N}_{2}\right]$ I. P. Natanson, Theory of functions of a real variable, Vol. II (translated by Leo F. Boron), Ungar, New York, 1960.

[R] H. Rubin, Representation of nonnegative continuous functions on product spaces, Bull. Amer. Math. Soc. 74 (1968), 332-333.

[W] S. Warner, The topology of compact convergence on continuous function spaces, Duke Math. J. 25 (1958), 265-282.

UNIVERSITY OF ROCHESTER 